\title{
From Anti-Severe Acute Respiratory Syndrome Coronavirus 2 Immune Response to Cancer Onset via Molecular Mimicry and Cross-Reactivity
}

\author{
Darja Kanduc ${ }^{1}$ \\ ${ }^{1}$ Department of Biosciences, Biotechnologies, and \\ Biopharmaceutics, University of Bari, Bari, Italy \\ Glob Med Genet 2021;8:176-182.
}

\begin{abstract}
Address for correspondence Darja Kanduc, PhD, Department of Biosciences, Biotechnologies, and Biopharmaceutics, University of Bari, Bari 70121, Italy (e-mail: dkanduc@gmail.com).
\end{abstract}

\author{
Abstract \\ Keywords \\ - SARS-CoV-2 spike gP \\ - tumor-suppressor \\ proteins \\ - molecular mimicry \\ - cross-reactivity \\ - long COVID \\ - cancer epidemic
}

Background and Objectives Whether exposure to severe acute respiratory syndrome coronavirus 2 (SARS-CoV-2) may predispose to the risk of cancer in individuals with no prior cancers is a crucial question that remains unclear. To confirm/refute possible relationships between exposure to the virus and ex novo insurgence of tumors, this study analyzed molecular mimicry and the related cross-reactive potential between SARS-CoV-2 spike glycoprotein (gp) antigen and human tumor-suppressor proteins. Materials and Methods Tumor-associated proteins were retrieved from UniProt database and analyzed for pentapeptide sharing with SARS-CoV-2 spike gp by using publicly available databases.

Results An impressively high level of molecular mimicry exists between SARS-CoV-2 spike gp and tumor-associated proteins. Numerically, 294 tumor-suppressor proteins share 308 pentapeptides with the viral antigen. Crucially, the shared peptides have a relevant immunologic potential by repeatedly occurring in experimentally validated epitopes. Such immunologic potential is of further relevancy in that most of the shared peptides are also present in infectious pathogens to which, in general, human population has already been exposed, thus indicating the possibility of immunologic imprint phenomena.

Conclusion This article described a vast peptide overlap between SARS-CoV-2 spike $\mathrm{gp}$ and tumor-suppressor proteins, and supports autoimmune cross-reactivity as a potential mechanism underlying prospective cancer insurgence following exposure to SARS-CoV-2. Clinically, the findings call for close surveillance of tumor sequelae that possibly could result from the current coronavirus pandemic.

\section{Introduction}

From lung damages to skin diseases and excessive immune responses, the disorders associated with severe acute respiratory syndrome coronavirus 2 (SARS-CoV-2), that is, coronavirus disease 2019 (COVID-19), are progressively being defined and diagnostically cataloged. ${ }^{1-7}$ Among the many

received

July 16,2021

accepted after revision

August 2, 2021

published online

September 7, 2021 diseases encompassed by COVID-19, clinical attention has focused on the relationship between SARS-CoV-2 and cancer. $^{8-10}$ Indeed, when compared with the pre-COVID-19 era, COVID-19 pandemic appears to be characterized by higher hospitalization and mortality rates in prostate cancer patients ${ }^{11}$; increased breast cancer dimensions ${ }^{12}$; increased proportion of patients with advanced non-small cell lung

\section{(c) 2021. The Author(s).}

This is an open access article published by Thieme under the terms of the Creative Commons Attribution License, permitting unrestricted use, distribution, and reproduction so long as the original work is properly cited. (https://creativecommons.org/licenses/by/4.0/)

Georg Thieme Verlag KG, Rüdigerstraße 14, 70469 Stuttgart, Germany 
cancer $^{13}$; and a higher number of diagnosed head and neck cancers (2.9-8.06\% in January-April 2020). ${ }^{14}$ Such data have been interpreted as due to the pressure exerted by the viral pandemic on the health care system, so cancer treatments have been delayed and, also, have been related to the viral infection per se. ${ }^{15}$ However, how SARS-CoV-2 infection might relate to cancer diseases remains unclear.

According to the research paradigm that peptide sequences common to pathogens and the human host may lead to autoimmunity through cross-reactivity, ${ }^{16-21}$ a previous report $^{22}$ has proposed cross-reactivity as a likely mechanism that can explain the immunopathology related to SARS-CoV2 exposure. As a matter of fact, many SARS-CoV-2-derived epitopes were shown to share peptide sequences with human proteins that are involved-when altered, mutated, deficient, and/or improperly functioning-in the etiology of the diseases encompassed by COVID-19. ${ }^{22}$ Moreover, and of special importance, it was noted that the viral versus human peptide sharing also involved human proteins related to pleuropulmonary blastoma, non-small cell lung cancer, breast invasive ductal carcinoma, multiple human cancers, tumor predisposition syndrome, and mesothelioma, inter alia. That is, the data suggested the possibility that morbidity/mortality increases in various tumors might represent long-term sequelae following exposure to SARS-CoV2 (Kanduc ${ }^{22}$ and pertinent references therein).

Hence, this study was undertaken to further explore the relationship between SARS-CoV-2 infection/active immunization and carcinogenesis, and specifically focused on the amino acid (aa) sequence identities between SARS-CoV-2 spike glycoprotein (gp) and tumor-suppressor human proteins. Analyses revealed a vast peptide sharing potentially able to generate pathogenic autoantibodies via cross-reactivity and immunologic imprinting phenomena, thus possibly leading to or enhancing the onset of a wide spectrum of cancer diseases.

\section{Materials and Methods}

Peptide sharing between SARS-CoV-2 spike gp (NCBI, GenBank Protein Accession ID $=$ QHD43416.1) and cancerrelated human proteins was analyzed using pentapeptides as sequence probes as already described. ${ }^{16-22}$ Pentapeptides were used as minimal immune determinant units since a peptide grouping formed of five aa residues defines an immune unit that can (1) induce highly specific antibodies and (2) determine antigen-antibody-specific interaction (Kanduc $^{23,24}$ and further references therein). Seven hundred eighty-two human proteins (in)directly linked to cancer were obtained from UniProtKB database (www.uniprot. org $)^{25}$ using "tumor suppressor" as keywords and are listed by UniProt entry in - Supplementary Table S1 (available in online version only)

Methodologically, the spike gp primary sequence was dissected into pentapeptides offset by one residue (i.e., MFVFL, FVFLV, VFLVL, FLVLL, and so forth) and the resulting viral pentapeptides were analyzed for occurrences within the human proteins related to cancer. The shared peptides were also controlled for occurrences in the pathogens Bor- detella pertussis, Corynebacterium diphtheriae, Clostridium tetani, Haemophilus influenzae, and Neisseria meningitides. The publicly available peptide match and peptide search programs (www.uniprot.org) were used. ${ }^{25}$

The immunologic potential of the peptides shared between SARS-CoV-2 spike gp and cancer-related proteins was investigated by searching the Immune Epitope Database (IEDB, www.iedb.org) ${ }^{26}$ for experimentally validated immunoreactive SARS-CoV-2 spike gp-derived epitopes hosting the shared pentapeptides.

\section{Results and Discussion}

Searching UniProt database for tumor-suppressor proteins produced 782 protein entries (in)directly related to tumorsuppressor activity and listed in - Supplementary Table S1 (available in online version only). Of the 782 proteins, 294 have pentapeptides in common with the spike gp, in a total of 308 occurrences (in all, 462, including multiple occurrences). These numbers certify the existence of an impressive, unexpected level of molecular mimicry between the viral antigen and the cancer-related human proteins. Obvious reasons of space prevent a detailed analysis peptide-by-peptide of the peptide overlap that is given in its entirety in - Supplementary Table S2 (available in online version only). Here in text, a snapshot of the peptide sharing is reported and discussed.

\section{Peptide Sharing between SARS-CoV-2 Spike gp and Tumor-Suppressor Proteins}

- Table 1 shows data relative to a representative sample of 19 tumor-suppressor proteins and documents that the peptide commonality with the viral antigen amounts to 29 pentapeptides. From a pathological perspective, - Table 1 clearly illustrates that even hitting only 19 out of the 294 tumorsuppressor proteins described in - Supplementary Table S2 (available in online version only) might equate to induce or enhance carcinogenesis in almost all of the human organs, from brain and liver to lung and bones. Examples of the cancers that might be evoked/potentiated by exposure to SARS-CoV-2 in the next future are $\mathrm{T}$ cell acute lymphoblastic leukemia, oligodendrogliomas, breast/ovarian cancers, sarcoma, malignant mesothelioma, B cell chronic lymphocytic leukemia, and non-small cell lung carcinoma, among the others.

\section{Immunologic Potential of the Peptide Sharing between SARS-CoV-2 Spike gP and Tumor-Suppressor Proteins}

The gloomy outlook hinted at by the findings described in - Table $\mathbf{1}$ becomes all the more likely in light of the high immunologic potential of the shared peptides. De facto, investigation of IEDB shows that the 29 pentapeptides shared by the spike gp antigen and the 19 tumor-suppressor proteins ( - Table 1 ) occur and recur in 150 epitopes derived from SARS-CoV-2 that have been experimentally validated and are cataloged as immunoreactive (-Table $\mathbf{2}$ ).

In essence, -Table $\mathbf{2}$ factually supports the possibility that cross-reactions can be triggered by SARS-CoV-2 infection/active immunization and hit human proteins 
Table 1 Peptide sharing between SARS-CoV-2 spike gp and a representative sample of 19 tumor-suppressor proteins

\begin{tabular}{|c|c|c|}
\hline Shared peptides & Tumor-suppressor proteins and related cancer diseases $^{a}$ & Refs $^{b}$ \\
\hline DPFLG & BC11B. B cell lymphoma/leukemia 11B. T cell acute lymphoblastic leukemia & 27,28 \\
\hline LPPLL, GAGAA, QDVVN, SPDVD & BICRA. Glioma tumor suppressor candidate. Oligodendrogliomas & 29 \\
\hline EPQII & BRCA1. Breast cancer type 1 susceptibility protein. Breast/ovarian cancer & 30 \\
\hline SLGAE, LAATK, EPVLK & BRCA2. Breast cancer type 2 susceptibility protein. Breast cancer & 31 \\
\hline RVVVL & DCC. Netrin receptor DCC. Deleted in colorectal carcinoma. Gallbladder cancer & 32 \\
\hline YRVVV, SALGK & $\begin{array}{l}\text { DIRA1. GTP-binding protein Di-Ras1. Small GTP-binding tumor suppressor } 1 . \\
\text { Lost/downregulated in neural tumors }\end{array}$ & 33 \\
\hline ITDAV & EXT1. Exostosin-1. Putative tumor suppressor protein EXT1. Bone tumors & 34 \\
\hline ALLAG & EXT2. Exostosin-2. Putative tumor suppressor protein EXT2. Bone tumors & 34 \\
\hline TLKSF, RLQSL & IL24. Interleukin-24. Suppression of tumorigenicity 16 protein. Melanoma & 35,36 \\
\hline SKPSK & LATS1. Large tumor suppressor homolog 1. Soft tissue sarcoma. & 37 \\
\hline ARDLI & LATS2. Large tumor suppressor homolog 2. Malignant mesothelioma & 38 \\
\hline YSNNS & $\begin{array}{l}\text { MTUS1. Microtubule-associated tumor suppressor 1. Hepatocellular } \\
\text { carcinoma }\end{array}$ & 39 \\
\hline GAGAA & PLAT2. Phospholipase A and acyltransferase 2. Gastric cancer & 40 \\
\hline GAGAA & PLAT3. Phospholipase A and acyltransferase 3. Ovarian carcinoma cells & 41 \\
\hline ADAGF, TYVPA & RBM5. Putative tumor suppressor LUCA15. Lung cancer & 42 \\
\hline RDLPQ, NSVAY & SCAI. Suppressor of cancer cell invasion. Downregulated in human tumors & 43 \\
\hline LLTDE & SDS3. Suppressor of defective silencing 3 protein homolog. Antitumor activity & 44 \\
\hline TQSLL, NFKNL, AGAAA & TASOR. Transcription activation suppressor. Clear cell renal cell carcinoma & 45 \\
\hline LSRLD, GDSSS & $\begin{array}{l}\text { TRI13. B cell chronic lymphocytic leukemia tumor suppressor Leu5. B cell } \\
\text { chronic lymphocytic leukemia. Non-small cell lung carcinoma }\end{array}$ & 46,47 \\
\hline
\end{tabular}

Abbreviations: gp, glycoprotein; SARS-CoV-2, severe acute respiratory syndrome coronavirus 2 .

${ }^{a}$ Tumor-suppressor proteins given by UniProt entry are in italic.

${ }^{\mathrm{b}}$ Further references on cancer diseases are available at UniProt, OMIM, and PubMed.

related to carcinogenesis. Very much this conclusion applies when considering that the extent of the potential immunologic cross-reactivity as well as the spectrum of potentially inducible tumors may be exponentially higher in light of the fact that $\boldsymbol{二}$ Tables $\mathbf{1}$ and $\mathbf{2}$ refer to the peptide commonality involving only a tiny part (19 out of 294) of the human proteins that-if altered-may lead to cancer (see - Supplementary Table S2 [available in online version only] for the peptide sharing in its totality).

\section{Potential Immunologic Imprint}

The 29 pentapeptides common to SARS-CoV-2 spike gp and tumor-suppressor proteins ( $\mathbf{-}$ Table $\mathbf{1}$ ) are not only present in immunoreactive epitopes ( - Table 2 ) but, in addition, almost all of them (24 out of 29) are also present in microbial organisms such as Bordetella pertussis, C. diphtheriae, $C$. tetani, $H$. influenzae, and $N$. meningitides ( - Table 3 ). That is, most of the shared peptides are also present in pathogens that an individual possibly encountered during his life because of infections and/or vaccinal routes.

Such interpathogen peptide commonality introduces the immunologic memory as a factor capable of enhancing the extent of the immune cross-reactive response against the tumor-suppressor proteins. That is, as already described since $1947,{ }^{48,49}$ the immune system does not induce ex novo primary responses toward a recent infection. Rather, the immune system recalls, amplifies, and intensifies preexisting memory responses toward past infections. In this way, what should have been a primary response to a recent infection is transformed into an anamnestic, secondary, and magnified response to past infections. Simply put, as already discussed in previous reports, ${ }^{50-55}$ the early history of the individual's infections/vaccinations dictates the immune outcomes of any successive infections/vaccinations.

The immunologic imprint phenomenon has its molecular foundations in the massive peptide sharing that characterizes microbial and human proteins ${ }^{17,56,57}$ and of which - Table 3 is an example. The implications are noteworthy. In the case object, following exposure to SARS-CoV2 by infection or vaccination, the expected primary response to the virus can turn into a secondary response to previously encountered pathogens against which the immune system already reacted and of which has stored an immunologic memory, that is, the microbial organisms reported in -Table $\mathbf{3}$. However, the previously encountered pathogens are no more present in the human organism, so that the anamnestic immune response triggered by the exposure to SARS-CoV-2 by infection or vaccination ends 
Table 2 Immunoreactive SARS-CoV-2 spike gp-derived epitopes containing peptides shared between SARS-CoV-2 spike gP and tumor-suppressor human proteins

\begin{tabular}{|c|c|c|c|}
\hline IEDB ID ${ }^{a}$ & Epitope sequence $^{\mathrm{b}}$ & IEDB ID $^{a}$ & Epitope sequence $^{\mathrm{b}}$ \\
\hline 36724 & litgRLQSL & 1329082 & ADAGFikqygdclgdia \\
\hline 38831 & IQDVVNqnaqalntl & 1329083 & ADAGFikqygdclgdiaa \\
\hline 51999 & qpYRVVVLsf & 1329254 & demiaqytsALLAG \\
\hline 54725 & RLQSLqtyv & 1329256 & demiaqytsALLAGt \\
\hline 533447 & raaeirasanLAATK & 1329258 & demiaqytsALLAGti \\
\hline 1069290 & cTLKSFtvekgiyqt & 1329260 & demiaqytsALLAGtit \\
\hline 1069445 & EPQIIttdntfvsgn & 1329323 & efqfenDPFLGvyy \\
\hline 1073938 & vqidrlitgRLQSLq & 1329325 & efqfenDPFLGvyyh \\
\hline 1074928 & ilpdpSKPSK & 1329327 & efqfenDPFLGvyyhk \\
\hline 1125063 & gltvLPPLL & 1329329 & efqfenDPFLGvyyhkn \\
\hline 1309132 & nfsqilpdpSKPSKr & 1329342 & emiaqytsALLAG \\
\hline 1309418 & aeirasanLAATKmsecvlg & 1329344 & emiaqytsALLAGt \\
\hline 1309447 & dfggfnfsqilpdpSKPSKr & 1329345 & emiaqytsALLAGtit \\
\hline 1309450 & dplsetkcTLKSFtvekgiy & 1329353 & EPQIIttdntfvsg \\
\hline 1309451 & dsfkeeldkyfknhtSPDVD & 1329390 & fcnDPFLGvyyh \\
\hline 1309467 & fdeddsEPVLKgvklhyt & 1329414 & fqfenDPFLGvyy \\
\hline 1309478 & gNFKNLrefvfknidgyfki & 1329416 & fafcnDPFLGvyyh \\
\hline 1309482 & gyqpYRVVVLsfellhapat & 1329422 & fsqilpdpSKPSKr \\
\hline 1309515 & IhrsyltpGDSSSgwtagaa & 1329571 & idrlitgRLQSLq \\
\hline 1309516 & litgRLQSLqtyvtqqlira & 1329572 & idrlitgRLQSLqt \\
\hline 1309519 & IpdpSKPSKrsfiedllfnk & 1329595 & iqdslsstaSALGKlq \\
\hline 1309523 & IssnfgaissvlndiLSRLD & 1329597 & iraaeirasanLAATK \\
\hline 1309532 & ngltvLPPLLTDEmiaqyts & 1329606 & ITDAVdcaldplse \\
\hline 1309534 & nitrfqTLLALhrsyltpgd & 1329627 & khtpinlvRDLPQg \\
\hline 1309546 & pflmdlegkqgNFKNLrefv & 1329659 & IADAGFikqygdclgdiaa \\
\hline 1309556 & qfenDPFLGvyyhknnkswm & 1329710 & IpdpSKPSKrsfiedllfnkvt \\
\hline 1309561 & qrnfyEPQIIttdntfvsgn & 1329762 & miaqytsALLAG \\
\hline 1309566 & qygdclgdiaARDLIcaqkf & 1329764 & miaqytsALLAGt \\
\hline 1309567 & RDLPQgfsaleplvdlpigi & 1329793 & ndiLSRLDkveaevq \\
\hline 1309585 & sssgwtAGAAAyyvgylqpr & 1329940 & qidrlitgRLQSLqt \\
\hline 1309589 & sygfqptngvgyqpYRVVVL & 1329966 & qpYRVVVLsfellhapa \\
\hline 1309593 & tITDAVdcaldplsetkctl & 1329969 & qsiiaytmSLGAE \\
\hline 1309599 & TYVPAqeknfttapaichdg & 1329978 & raaeirasanLAATKm \\
\hline 1309605 & vsngthwfvtqrnfyEPQII & 1330138 & staSALGKIQDVVN \\
\hline 1310254 & aeNSVAYSNNSiaip & 1330167 & tdemiaqytsALLAGt \\
\hline 1310284 & ARDLlcaqkfngltv & 1330169 & tdemiaqytsALLAGti \\
\hline 1310303 & caqkfngltvLPPLL & 1330171 & tdemiaqytsALLAGtit \\
\hline 1310362 & eldkyfknhtSPDVD & 1330209 & TLKSFtvekgiyqts \\
\hline 1310392 & fgttldskTQSLLiv & 1330210 & TLKSFtvekgiyqtsn \\
\hline 1310415 & fngltvLPPLLTDEm & 1330211 & TLKSFtvekgiyqtsnf \\
\hline 1310448 & gkIQDVVNqnaqaln & 1330219 & tpGDSSSgwtAGAAA \\
\hline 1310586 & litgRLQSLqtyvtq & 1330220 & tpinlvRDLPQg \\
\hline 1310609 & IpdpSKPSKrsfied & 1330305 & vqidrlitgRLQSLqt \\
\hline
\end{tabular}


Table 2 (Continued)

\begin{tabular}{|c|c|c|c|}
\hline IEDB ID ${ }^{a}$ & Epitope sequence $^{\mathrm{b}}$ & IEDB ID $^{\mathrm{a}}$ & Epitope sequence $^{\mathrm{b}}$ \\
\hline 1310611 & LPPLLTDEmiaqyts & 1330306 & vqidrlitgRLQSLqtyv \\
\hline 1310747 & qpYRVVVLsfellha & 1330368 & yfkiyskhtpinlvRDLPQ \\
\hline 1310750 & qrnfyEPQIIttdnt & 1330391 & ytsALLAGtit \\
\hline 1310847 & titsgwtfGAGAAlq & 1330433 & diLSRLD \\
\hline 1310947 & wtfGAGAAlqipfam & 1330434 & diLSRLDppeaevq \\
\hline 1311657 & ccscgscckfdeddsEPVLKgvkl & 1330437 & dslsstaSALGKI \\
\hline 1311782 & pdpSKPSKrsfiedllfnkvtlad & 1330438 & dslsstaSALGKlq \\
\hline 1312257 & cckfdeddsEPVLKg & 1330439 & dsIsstaSALGKIqdv \\
\hline 1312283 & deddsEPVLKgvklh & 1330447 & EPQIIttdntfvsgnc \\
\hline 1312733 & ilpdpSKPSKrsfie & 1330456 & fsqilpdpSKPSK \\
\hline 1312780 & ITDAVdcaldplset & 1330457 & fsqilpdpSKPSKrs \\
\hline 1313154 & miaqytsALLAGtit & 1330463 & gfnfsqilpdpSKPSKr \\
\hline 1313286 & pinlvRDLPQgfwal & 1330487 & ilpdpSKPSKr \\
\hline 1313756 & TLKSFtvek & 1330489 & iqdsIsstaSALGKI \\
\hline 1313930 & vTYVPAqeknfttap & 1330490 & iqdsIsstaSALGKlqd \\
\hline 1314170 & ADAGFikqy & 1330515 & IADAGFikqy \\
\hline 1315180 & aYSNNSiai & 1330551 & pSKPSKrsf \\
\hline 1316068 & etkcTLKSF & 1330552 & pSKPSKrsfi \\
\hline 1316945 & fsqilpdpSKPSKrsfie & 1330557 & qilpdpSKPSKr \\
\hline 1318209 & hvTYVPAqek & 1330589 & slsstaSALGKlq \\
\hline 1320443 & IgaeNSVAY & 1330597 & sqilpdpSKPSK \\
\hline 1321084 & LPPLLTDEm & 1330598 & sqilpdpSKPSKr \\
\hline 1323467 & qpYRVVVL & 1330623 & tpinlvRDLPQgfs \\
\hline 1323750 & rasanLAATK & 1330624 & tpinlvRDLPQgfsa \\
\hline 1323919 & RLQSLqty & 1330625 & tpinlvRDLPQgfsalepl \\
\hline 1324353 & setkcTLKSF & 1331139 & cnDPFLGvy \\
\hline 1325536 & tIADAGFik & 1332424 & itgRLQSLqty \\
\hline 1327824 & wtAGAAAyy & 1332664 & LLTDEmiaqy \\
\hline 1327836 & wtfGAGAAI & 1334122 & TYVPAqeknft \\
\hline 1328800 & ytmslgaeNSVAY & 1334394 & yqpYRVVVL \\
\hline 1328800 & ytmSLGAEnsvay & 1334452 & alhrsyltpGDSSSg \\
\hline 1329076 & aaeirasanLAATK & 1334473 & NSVAYSNNSiaiptnft \\
\hline
\end{tabular}

Abbreviations: gp, glycoprotein; IEDB, Immune Epitope Database; SARS-CoV-2, severe acute respiratory syndrome coronavirus 2.

aEpitopes listed according to the IEDB ID number.

bShared sequences given are capitalized.

to divert onto available immune determinants that, in the present case, are the common determinants present in the tumor-suppressor human proteins. Pathologically, one has to consider that usually an anamnestic secondary immune response is characterized by high avidity and high affinity, besides being quantitatively relevant. Therefore, as a final result, exposure to SARS-CoV-2 by infection and/or vaccination can trigger immediate and violent cross-reactive attacks against the proteins that protect the human being from carcinogenesis.

\section{Conclusion}

The findings described in - Tables 1 to 3 and - Supplementary Table S2 (available in online version only) indicate that molecular mimicry and cross-reactivity between peptides common to SARS-CoV-2 and tumor-related proteins might cause/contribute to cancer epidemics worldwide in the next future. The potential cancer risk might be enhanced by immunologic imprinting phenomena, given the fact that the comparative analyses shown in - Table 3 indicate the possibility 
Table 3 Occurrences in microbial organisms of pentapeptides common to SARS-CoV-2 spike gp, human proteins related to cancer, and SARS-CoV-2 spike gp-derived epitopes

\begin{tabular}{|l|l|}
\hline Organism & Shared peptides \\
\hline Bordetella pertussis & ADAGF, AGAAA, ALLAG, GAGAA, ITDAV, RLQSL, SLGAE, SPDVD, TYVPA \\
\hline Clostridium tetani & AGAAA, LAATK, LLTDE, YSNNS \\
\hline Corynebacterium diphtheriae & AGAAA, ALLAG, EPQII, GAGAA, ITDAV, SALGK, YRVVV \\
\hline Haemophilus influenzae & AGAAA, GAGAA, LLTDE, LPPLL, LSRLD, NFKNL, NSVAY, RDLPQ, \\
& RLQSL, RVVVL, SALGK, SLGAE, TLKSF, TQSLL, YSNNS \\
\hline Neisseria meningitides & AGAAA, ALLAG, EPVLK, GAGAA, LLTDE, LPPLL \\
\hline
\end{tabular}

Abbreviations: gp, glycoprotein; SARS-CoV-2, severe acute respiratory syndrome coronavirus 2.

that a preexisting immune response to previously encountered pathogens could be magnified and intensified following SARSCoV-2 infection/active immunization. These data are disturbing and invite to immediately intensify clinical surveillance in oncology and to undertake rigid cancer prevention actions, including healthy lifestyle and continuous controls. It will be vital to formulate/implement actions that contemplate fast and safe procedures for clinical trials, development of specific and reliable tumor markers for diagnosis, accurate follow-up of treatments, and, administratively, medical health records, detailed registries, biobanks, health surveys, and coordinated observational studies. Never before do all the recommendations of the European plan for the fight against cancer appear current and necessary. ${ }^{58}$ De facto, tumors appear to be the predominant pathologies that will populate the post pandemic long COVID-19.

\section{Funding}

None.

\section{Conflict of Interest}

None declared.

\section{References}

1 Huang C, Wang Y, Li X, et al. Clinical features of patients infected with 2019 novel coronavirus in Wuhan, China. Lancet 2020;395 (10223):497-506

2 Sadeghzadeh-Bazargan A, Rezai M, Najar Nobari N, Mozafarpoor S, Goodarzi A. Skin manifestations as potential symptoms of diffuse vascular injury in critical COVID-19 patients. J Cutan Pathol 2021. Doi: 10.1111/cup.14059

3 Boechat JL, Chora I, Morais A, Delgado L. The immune response to SARS-CoV-2 and COVID-19 immunopathology - current perspectives. Pulmonology 2021;27(05):423-437

4 Cabrera Martimbianco AL, Pacheco RL, Bagattini ÂM, Riera R. Frequency, signs and symptoms, and criteria adopted for long COVID-19: s systematic review. Int J Clin Pract 2021:e14357

5 Rosen HR, O'Connell C, Nadim MK, et al. Extrapulmonary manifestations of severe acute respiratory syndrome coronavirus-2 infection. J Med Virol 2021;93(05):2645-2653

6 Ramos-Casals M, Brito-Zerón P, Mariette X. Systemic and organspecific immune-related manifestations of COVID-19. Nat Rev Rheumatol 2021;17(06):315-332

7 Suárez-Reyes A, Villegas-Valverde CA. Implications of low-grade inflammation in SARS-CoV-2 immunopathology. MEDICC Rev 2021;23(02):42
8 Han HJ, Nwagwu C, Anyim O, Ekweremadu C, Kim S. COVID-19 and cancer: from basic mechanisms to vaccine development using nanotechnology. Int Immunopharmacol 2021;90:107247

9 Corti C, Curigliano G. Commentary: SARS-CoV-2 vaccines and cancer patients. Ann Oncol 2021;32(04):569-571

10 Xia P, Dubrovska A. Tumor markers as an entry for SARS-CoV-2 infection? FEBS J 2020;287(17):3677-3680

11 Chakravarty D, Ratnani P, Sobotka S, et al. Increased hospitalization and mortality from COVID-19 in prostate cancer patients. Cancers (Basel) 2021;13(07):1630

12 Vanni G, Pellicciaro M, Materazzo M, et al. Advanced stages and increased need for adjuvant treatments in breast cancer patients: the effect of the one-year COVID-19 pandemic. Anticancer Res 2021;41(05):2689-2696

13 Park JY, Lee YJ, Kim T, et al. Collateral effects of the coronavirus disease 2019 pandemic on lung cancer diagnosis in Korea. BMC Cancer 2020;20(01):1040

14 Taylor R, Omakobia E, Sood S, Glore RJ. The impact of coronavirus disease 2019 on head and neck cancer services: a UK tertiary centre study. J Laryngol Otol 2020;134(08):684-687

15 Derosa L, Melenotte C, Griscelli F, et al. The immuno-oncological challenge of COVID-19. Nat Can 2020;1:946-964

16 Natale C, Giannini T, Lucchese A, Kanduc D. Computer-assisted analysis of molecular mimicry between human papillomavirus 16 E7 oncoprotein and human protein sequences. Immunol Cell Biol 2000;78(06):580-585

17 Kanduc D, Stufano A, Lucchese G, Kusalik A. Massive peptide sharing between viral and human proteomes. Peptides 2008;29 (10):1755-1766

18 Lucchese G, Capone G, Kanduc D. Peptide sharing between influenza A H1N1 hemagglutinin and human axon guidance proteins. Schizophr Bull 2014;40(02):362-375

19 Kanduc D. Peptide cross-reactivity: the original sin of vaccines. Front Biosci (Schol Ed) 2012;4:1393-1401

20 Lucchese G, Kanduc D. Potential crossreactivity of human immune responses against HCMV glycoprotein B. Curr Drug Discov Technol 2016;13(01):16-24

21 Lucchese G, Kanduc D. Zika virus and autoimmunity: from microcephaly to Guillain-Barré syndrome, and beyond. Autoimmun Rev 2016;15(08):801-808

22 Kanduc D. From anti-SARS-CoV-2 immune responses to COVID19 via molecular mimicry. Antibodies (Basel) 2020;9(03):33

23 Kanduc D. Pentapeptides as minimal functional units in cell biology and immunology. Curr Protein Pept Sci 2013;14(02): $111-120$

24 Kanduc D. Homology, similarity, and identity in peptide epitope immunodefinition. J Pept Sci 2012;18(08):487-494

25 UniProt Consortium. UniProt: a worldwide hub of protein knowledge. Nucleic Acids Res 2019;47(D1):D506-D515

26 Vita R, Mahajan S, Overton JA, et al. The Immune Epitope Database (IEDB): 2018 update. Nucleic Acids Res 2019;47(D1):D339-D343 
27 Gutierrez A, Kentsis A, Sanda T, et al. The BCL11B tumor suppressor is mutated across the major molecular subtypes of T-cell acute lymphoblastic leukemia. Blood 2011;118(15):4169-4173

28 Bartram I, Gökbuget N, Schlee C, et al. Low expression of T-cell transcription factor BCL11b predicts inferior survival in adult standard risk T-cell acute lymphoblastic leukemia patients. J Hematol Oncol 2014;7:51

29 Yang P, Kollmeyer TM, Buckner K, Bamlet W, Ballman KV, Jenkins RB. Polymorphisms in GLTSCR1 and ERCC2 are associated with the development of oligodendrogliomas. Cancer 2005;103(11): 2363-2372

30 Clark SL, Rodriguez AM, Snyder RR, Hankins GD, Boehning D. Structure-function of the tumor suppressor BRCA1. Comput Struct Biotechnol J 2012;1(01):e201204005

31 Angeli D, Salvi S, Tedaldi G. Genetic predisposition to breast and ovarian cancers: how many and which genes to test? Int J Mol Sci 2020;21(03):1128

32 Rai R, Sharma KL, Tiwari S, Misra S, Kumar A, Mittal B. DCC (deleted in colorectal carcinoma) gene variants confer increased susceptibility to gallbladder cancer (Ref. No.: Gene-D-12-01446). Gene 2013;518(02):303-309

33 Ellis CA, Vos MD, Howell H, Vallecorsa T, Fults DW, Clark GJ. Rig is a novel Ras-related protein and potential neural tumor suppressor. Proc Natl Acad Sci U S A 2002;99(15):9876-9881

34 Duncan G, McCormick C, Tufaro F. The link between heparan sulfate and hereditary bone disease: finding a function for the EXT family of putative tumor suppressor proteins. J Clin Invest 2001;108(04):511-516

35 Huang EY, Madireddi MT, Gopalkrishnan RV, et al. Genomic structure, chromosomal localization and expression profile of a novel melanoma differentiation associated (mda-7) gene with cancer specific growth suppressing and apoptosis inducing properties. Oncogene 2001;20(48):7051-7063

36 Allen M, Pratscher B, Roka F, et al. Loss of novel mda-7 splice variant (mda-7s) expression is associated with metastatic melanoma. J Invest Dermatol 2004;123(03):583-588

37 Hisaoka M, Tanaka A, Hashimoto H. Molecular alterations of h-warts/LATS1 tumor suppressor in human soft tissue sarcoma. Lab Invest 2002;82(10):1427-1435

38 Murakami H, Mizuno T, Taniguchi T, et al. LATS2 is a tumor suppressor gene of malignant mesothelioma. Cancer Res 2011; 71(03):873-883

39 Di Benedetto M, Pineau P, Nouet S, et al. Mutation analysis of the $8 \mathrm{p} 22$ candidate tumor suppressor gene ATIP/MTUS1 in hepatocellular carcinoma. Mol Cell Endocrinol 2006;252 (1-2):207-215

40 Liang Y, Zhang C, Dai DQ. Identification of DNA methylationregulated differentially-expressed genes and related pathways using Illumina $450 \mathrm{~K}$ BeadChip and bioinformatic analysis in gastric cancer. Pathol Res Pract 2019;215(10):152570

41 Nazarenko I, Schäfer R, Sers C. Mechanisms of the HRSL3 tumor suppressor function in ovarian carcinoma cells. J Cell Sci 2007;120 (Pt 8):1393-1404

42 Oh JJ, Razfar A, Delgado I, et al. 3p21.3 tumor suppressor gene H37/Luca15/RBM5 inhibits growth of human lung cancer cells through cell cycle arrest and apoptosis. Cancer Res 2006;66(07): 3419-3427

43 Brandt DT, Baarlink C, Kitzing TM, et al. SCAI acts as a suppressor of cancer cell invasion through the transcriptional control of beta1-integrin. Nat Cell Biol 2009;11(05):557-568

44 Ramakrishna S, Suresh B, Bae SM, Ahn WS, Lim KH, Baek KH. Hyaluronan binding motifs of USP17 and SDS3 exhibit anti-tumor activity. PLoS One 2012;7(05):e37772

45 Iacobas DA, Mgbemena VE, Iacobas S, Menezes KM, Wang $\mathrm{H}$ Saganti PB. Genomic fabric remodeling in metastatic clear cell renal cell carcinoma (ccRCC): a new paradigm and proposal for a personalized gene therapy approach. Cancers (Basel) 2020;12 (12):3678

46 van Everdink WJ, Baranova A, Lummen C, et al. RFP2, c130RF1, and FAM10A4 are the most likely tumor suppressor gene candidates for B-cell chronic lymphocytic leukemia. Cancer Genet Cytogenet 2003;146(01):48-57

47 Xu L, Wu Q Zhou X, Wu Q, Fang M. TRIM13 inhibited cell proliferation and induced cell apoptosis by regulating NF-кB pathway in non-small-cell lung carcinoma cells. Gene 2019; 715:144015

48 Francis T, Salk JE, Quilligan JJ. Experience with vaccination against influenza in the spring of 1947: a preliminary report. Am J Public Health Nations Health 1947;37(08):1013-1016

49 Davenport FM, Hennessy AV, Francis T Jr. Epidemiologic and immunologic significance of age distribution of antibody to antigenic variants of influenza virus. J Exp Med 1953;98(06): 641-656

50 Lucchese G, Kanduc D. The Guillain-Barrè peptide signatures: from Zika virus to Campylobacter, and beyond. Virus Adaptation and Treatment 2017;9:1-11

51 Lucchese G, Kanduc D. Minimal immune determinants connect Zika virus, human cytomegalovirus, and Toxoplasma gondii to microcephaly-related human proteins. Am J Reprod Immunol 2017;77(02):e12608

52 Kanduc D. Immunobiology: on the inexistence of a negative selection process. Adv Stud Biol 2020;12(01):19-28

53 Kanduc D. Anti-SARS-CoV-2 Immune response and sudden death: titin as a link. Adv Stud Biol 2021;13(01):37-44

54 Kanduc D, Shoenfeld Y. Inter-pathogen peptide sharing and the original antigenic sin: solving a paradox. Open Immunol J 2018; 8:16-27

55 Kanduc D. Thromboses and hemostasis disorders associated with coronavirus disease 2019: the possible causal role of crossreactivity and immunological imprinting. Glob Med Genet 2021. Doi: $10.1055 / \mathrm{s}-0041-1731068$

56 Trost B, Lucchese G, Stufano A, Bickis M, Kusalik A, Kanduc D. No human protein is exempt from bacterial motifs, not even one. Self Nonself 2010;1(04):328-334

57 Kanduc D. The comparative biochemistry of viruses and humans: an evolutionary path towards autoimmunity. Biol Chem 2019; 400(05):629-638

58 Europe's Beating Cancer Plan: A new EU approach to prevention, treatment and care. [Internet] Accessed on May 2021 at: https:// ec.europa.eu/commission/presscorner/detail/en/ip_21_342 